\title{
Recent use of medical infrared thermography in skin neoplasms
}

\author{
\begin{tabular}{l|l|l} 
C. Magalhaes & 1 & R. Vardasca \\
& (D) | Jendes &
\end{tabular}
}

${ }^{1}$ Faculdade de Engenharia, Universidade do Porto, Porto, Portugal

${ }^{2}$ LABIOMEP, INEGI -LAETA, Faculdade de Engenharia, Universidade do Porto, Porto, Portugal

\section{Correspondence}

R. Vardasca, LABIOMEP, INEGI -LAETA, Faculdade de Engenharia, Universidade do Porto, Porto, Portugal.

Email: rvardasca@fe.up.pt

\section{Funding information} NORTE2020, Grant/Award Number: NORTE-01-0145-FEDER-000022; Fundação para a Ciência e a Tecnologia, Grant/Award Number: PEst-OE/EME/LA0022/2013

\begin{abstract}
Background: Infrared thermal imaging captures the infrared radiation emitted by the skin surface. The thermograms contain valuable information, since the temperature distribution can be used to characterize physiological anomalies. Thus, the use of infrared thermal imaging (IRT) has been studied as a possible medical tool to aid in the diagnosis of skin oncological lesions. The aim of this review is to assess the current state of the applications of IRT in skin neoplasm identification and characterization. Methods: A literature survey was conducted using the reference bibliographic databases: Scopus, PubMed and ISI Web of Science. Keywords (thermography, infrared imaging, thermal imaging and skin cancer) were combined and its presence was verified at the title and abstract of the article or as a main topic. Only articles published after 2013 were considered during this search.

Results: In total, 55 articles were encountered, resulting in 14 publications for revision after applying the exclusion criteria. It was denoted that IRT have been used to characterize and distinguish between malignant and benign neoplasms and different skin cancer types. IRT has also been successfully applied in the treatment evaluation of these types of lesions.

Conclusion: Trends and future challenges have been established to improve the application of IRT in this field, disclosing that dynamic thermography is a promising tool for early identification of oncological skin conditions.
\end{abstract}

\section{KEYWORDS}

dynamic thermal imaging, medical diagnostic information, skin cancer, steady-state thermal imaging

\section{1 | INTRODUCTION}

Over the last years, skin cancer incidence has risen to a worrying level, as a consequence of risky behaviours that people are subject to, especially excessive exposure to UV radiation. As the mortality rates associated with these tumours decrease with the early detection, a precise diagnosis is imperative to prevent unnecessary complications. ${ }^{1,2}$ Thermoregulation is one of the main functions of the human skin and is primarily controlled by vasculature alterations that occur when the body is subjected to external or internal stimulus, for example, heat exposure and alterations in the carbon dioxide level respectively. ${ }^{3}$ This particular characteristic has been used for ages as a diagnosis aid, since skin temperature impairments often indicate health issues. Therefore, methods that take advantage of this feature, such as infrared thermal (IRT) imaging, have been explored for new applications in the medical field. Thermography, or IRT, is a contactless sensing method that uses a thermal camera to record the infrared radiation that is emitted by the skin, involving no health hazard for the patient, due to the absence of ionizing radiation. The temperature distribution of the surface under analysis is displayed in a thermogram, providing physiological information that can be used to detect temperature abnormalities, like the ones encountered between a skin neoplasm and the healthy tissue that surrounds it. ${ }^{4-6}$ In addition, this tool can be applied dynamically, that is, with a thermal 
stimulus prior to the image acquisition process, ${ }^{7}$ with the goal of increasing the temperature differences between the lesion and the surrounding skin, or in a steady-state, without any heat stress. Thus, several researchers have studied the use of dynamic infrared in skin neoplasms, as a complementary tool in the diagnosis process, to improve the accuracy of the procedure, decreasing, simultaneously, the stress and discomfort of the patient. ${ }^{8-10}$ This systematic review aims to ascertain the current state of the applications of medical thermography in skin neoplasms, as well as understand possible challenges that can arise during this process, establishing improvements for future studies conducted in this area.

\section{2 | MATERIAL AND METHODS}

\section{1 | Search strategy}

The literature research was conducted, using the following combination of keywords, in the bibliographic databases: Scopus, PubMed and ISI Web of Science respectively: ((TITLE-ABS-KEY (skin cancer) AND TITLE-ABS-KEY (thermography OR (infrared imaging) OR (thermal imaging)))); ((skin cancer [Title/Abstract]) AND (thermography[Title/Abstract] OR (infrared imaging[Title/ Abstract]) OR (thermal imaging[Title/Abstract])); TOPIC: (skin cancer) AND TOPIC: (thermography OR (infrared imaging) OR (thermal imaging)). The terms used for the search were clear and basic, to increase the number of results encountered, and the field selection applied in each reference source was used to guarantee the consistency of the research. The Boolean operator OR was included in the search, since "infrared imaging" and "thermal imaging" are often used as synonyms of "thermography." Only publications with dates from 1 January 2014 to the date of this research, that is, 31 March 2017 were included. A duplicate removal was performed at the end.

\section{2 | Screening and eligibility results}

The title and abstracts of the encountered publications were, firstly, analysed, including only the articles that referred the use of medical thermography for the evaluation of skin neoplasms.

The first eligibility criterion involved the elimination of articles that reported the use of IRT imaging in skin cancer cells, instead of the neoplasm itself. Additionally, meeting abstracts and revision articles were eliminated, making the second criterion. The third selection parameter consisted of keeping the articles written in English and excluding publications in other languages. Considering that this review is focused in a single imaging modality, that is, thermographic, some articles based on the use of infrared spectral imaging, encountered due to the terms used in the bibliographic research, were removed, making it the fourth eligibility criteria. Finally, publications focused on the description of thermal technology used for the detection of skin cancer, were eliminated from the remaining results.

The final set of articles was then separated into three main classes: clinical studies for diagnosis applications, treatment monitoring in clinical situations and theoretical studies, for a full-text review. The entire process was performed taking into account the PRISMA rules for systematic reviews described in Refs. (11,12). Figure 1 summarizing the phases of this revision process.

\section{3 | RESULTS}

The conducted bibliographic search resulted in a total of 51 publications, being 10, 9 and 32 encountered in ISI Web of Knowledge, PubMed and Scopus respectively. Four additional records were identified through Google Scholar, being included in the literature search results. After duplicate removal, 29
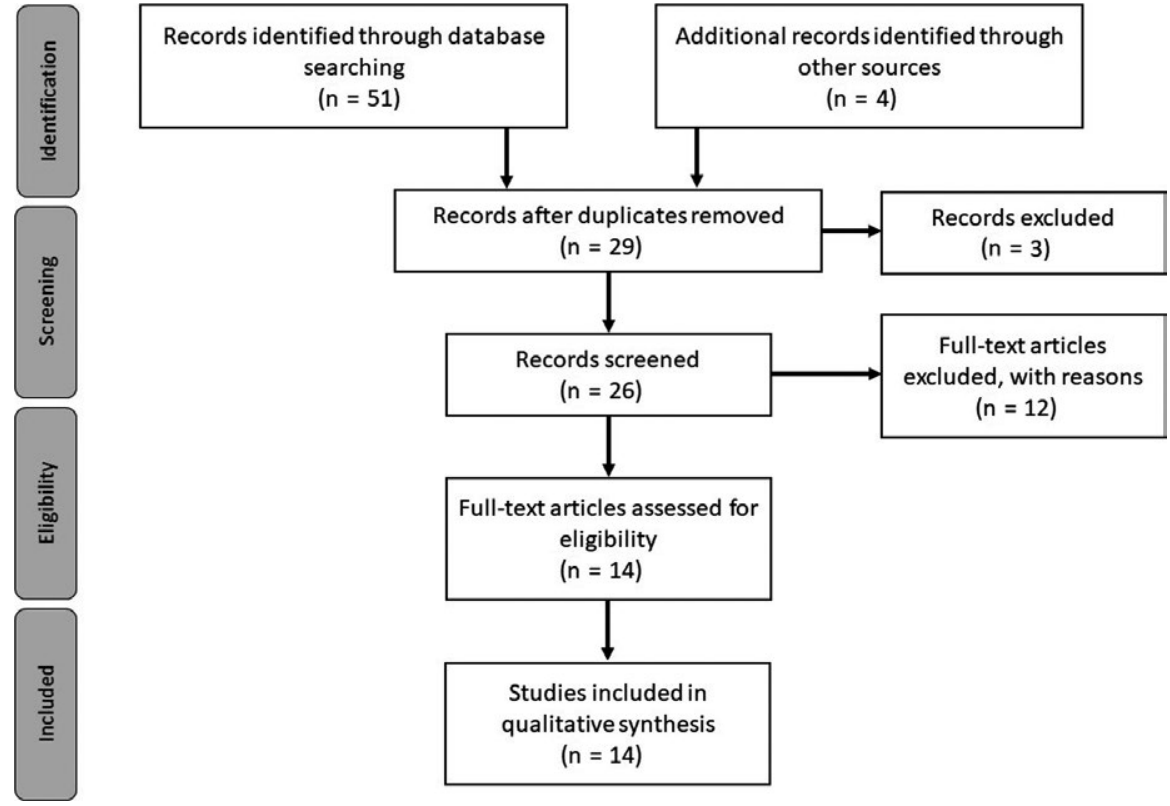

FIGURE 1 PRISMA flow diagram. Adapted from Moher et al ${ }^{12}$ 
articles were kept. The abstract and title screening resulted in the exclusion of 3 articles, since no application of medical thermography to skin neoplasms was studied in the publication. The eligibility criteria previously mentioned lead to the elimination of 12 articles, being the first, second, third and fourth criterion responsible for the exclusion of $1,3,3$ and 5 publications respectively. Thus, 14 full-text articles remained for revision, 6 articles describe the use of IRT as a diagnosis support tool in clinical applications, 3 concern the use of thermography for skin neoplasms treatment screening in clinical studies and 5 are included in the theoretical studies category, for diagnosis purposes.

\section{1 | Diagnosis support method-clinical studies}

Concerning the use of IRT for the detection and identification of skin lesions, consent is verified in all the articles, indicating that thermography, applied with all its variants, has the possibility to be an effective complementary diagnostic support tool. The differences in temperature encountered between lesion areas and the surrounding tissue proved to be useful in the distinction between malignant and benign skin neoplasms. ${ }^{13,14}$ Godoy et al ${ }^{13}$ evidenced these results, using dynamic thermal imaging (DTI), with the recordings of the thermal recovery of skin tumours, after the application of a cold stress, achieving sensitivity and specificity values of $95 \%$ an $83 \%$ respectively.

The characterization of distinct types of skin lesions using IRT, has also been successfully studied, with the goal of providing more information to the practitioner during the diagnosis process, either by a steady-state ${ }^{15}$ or a dynamic ${ }^{16}$ process. A common idea is denoted by Di Carlo et al, ${ }^{16}$ with respect to the thermal patterns presented by the neoplasms, referring that, in general, malignant lesions are characterized by hyperthermic patterns, while the benign ones, present lower emissivity values. The use of video thermography allowed the complete separation (100\%) between basal cell carcinomas (BCC) and actinic keratosis (AK) lesions, having the first type of skin neoplasm displayed a hypothermic halo and the latter a hyperthermic one. ${ }^{16}$ The work of Stringasci et al ${ }^{15}$ with steady-state thermography, reinforced, by and large, these findings, having BCC showed lower emissivity values and intradermal naevus lower temperatures than the basal type. The distinction between squamous cell carcinoma (SCC), which showed an hyperthermic pattern, and $\mathrm{AK}$, with temperature values similar to the skin, was also successfully carried out.

Infrared thermal imaging proved to be a secure tool to aid in the detection of melanoma ${ }^{17,18}$ with high specificity, even though it presents varying sensitivity values, depending, partly, on the equipment used. Solivetti et al ${ }^{17}$ showed that telethermography, associated with thermo-stimulation, allows the detection of melanoma skin cancer without false positives, avoiding discomfort and stress for patients with unnecessary biopsies. Moreover, in a distinct study, ${ }^{18}$ DTI was able to detect $60.87 \%$ of early melanomas, showing the potential of this tool for precocious diagnosis.

\section{2 | Treatment monitoring-clinical studies}

Apart from diagnosis, medical thermography has demonstrated its usefulness on the patient follow-up, through the evaluation of skin lesions, such as $\mathrm{AK}^{19}$ and melanoma, ${ }^{20}$ after treatment. Laino et $\mathrm{al}^{19}$ used active telethermography to evaluate the reduction in the hyperthermic halo of $\mathrm{AK}$, after its removal and lesion site treatment with Eryfotona. Eryfotona. IRT also helped in the temperature monitoring of malignant melanoma, during electrical stimulation therapy via liquid metal printed electronics on mice skin, as seen in, ${ }^{20}$ showing no alterations, in the thermal pattern of the lesion site, during this step.

Additionally, steady-state thermographic measurements have been used to verify the changes that occur, in the skin temperature gradient, of BCC, during treatment with photodynamic therapy, ${ }^{21}$ having Cholewka et al verified that some temperature changes in the place of injury might be caused by the treatment itself and need to be considered in the conclusions drawn.

\section{3 | Theoretical studies}

Infrared thermal imaging has shown its potential, in theoretical studies, for the detection of melanoma, in distinct phases of development. Bonmarin and Le $\mathrm{Gal}^{22}$ constructed a computational model that emulated different skin cancer stages, and tested the ability of steady-state and transient-state-lock-in thermal imaging (LIT)-thermography in the detection of such tumours. The phase images, retrieved from the demodulation of the transient skin surface temperature signals, seem promising for the detection of such precocious lesions. Another mathematical model, developed by Bhowmik et $a^{23}$ studied the use of dynamic infrared imaging, in 4 different melanoma stages, that is, early stage (ES I), Clark II (CL II), III (CL III) and IV (CL IV), for its detection and establishment of criteria for the early diagnosis. Even though it was shown that IRT evaluation of stage I melanoma was achievable, with an accurate analysis of the thermograms, the presented model was not fully efficient in the detection of early stage melanoma. In a more recent study, the same author ${ }^{24}$ proposed the use of frequency modulated thermal wave imaging, for the detection of the abovementioned stages, in a $3 \mathrm{D}$ computational model. The active thermal imaging method proposed, displayed a low efficiency in the detection of ES I and $C L I$ I, being its identification only possible, when combined with the phase images acquired from the thermal signals. Steady-state thermography was also successfully used to distinguish between benign lesions and melanomas with no vasculature, ${ }^{25}$ depending on the metabolic rate and tumour size selected for the theoretical model.

Cheng and Herman ${ }^{26}$ used DTI, in a 2D skin surface model, to study the most suited cooling method and parameters for the detection of early stages melanoma. The optimal conditions included constant cooling at $20^{\circ} \mathrm{C}$ for a period of 2 minutes that can be adjusted according to the stage of the lesion under evaluation. 


\section{4 | DISCUSSION OF TRENDS AND FUTURE CHALLENGES}

For clinical studies, the increase in the patient population number is one of the main trends appointed for future work, by some authors, ${ }^{13,16,17,21}$ in order to confirm the validity of the presented methods and attain extensive statistical analysis. The development of new image acquisition protocols is also of concern, due to the common presence of hair, that affects the thermal measurements, and the need of patient immobility, during the image acquisition process, which can be uncomfortable for the subject under test. ${ }^{13}$

Further research, focusing on the development of new diagnosis algorithms, for the analysis and processing of the thermal images, are needed, to improve the final results. ${ }^{15}$ It is of value to exploit, in a more detailed way, the physiology of skin neoplasms, to better understand the differences in temperature, between the lesion site and the surrounding healthy skin, during the thermal recovery. ${ }^{13}$ Additionally, the definition of rates of specificity and sensitivity of the thermal information, retrieved from the studies' results, are also of importance. ${ }^{15}$

Concerning theoretical approaches, the construction or refinement of 3D skin lesion computational models is necessary, in order to resemble, as much as possible, with a real biological model. ${ }^{25}$ Upcoming research, related to the study of different modulation frequencies in LIT is appointed as necessary for the diagnosis of stage I and II melanoma. ${ }^{22}$ Moreover, the investigation of different tumour sizes, locations and varying blood vessel sizes could also be of interest, for future work. ${ }^{23,25}$

The use of IRT dynamically appears to be a rising tendency in skin cancer studies, ${ }^{13,16-19,22-24,26}$ as a mean of improving thermal patterns, facilitating the detection and characterization of different skin cancer types.

\section{5 | CONCLUSION}

Infrared thermal imaging is a non-invasive, inexpensive imaging modality that is easy to perform, taking advantage of thermal cameras to detect the infrared radiation emitted by the skin, to originate thermal patterns characteristic of a specific body region. It has been used not only as an assistive tool for the diagnosis of skin cancer lesions, such as, melanoma, BCC, SCC and AK but also for skin lesion monitoring after treatment. Even though, its success has been reported in the identification of skin cancer, theoretical studies indicate that early stage melanoma identification is still not achievable. Several improvements for future work have been noted with the goal of developing this technique to its full potential, particularly its dynamic application, increasing the quality of the medical services provided to the patients, in skin cancer-related procedures.

\section{ACKNOWLEDGEMENTS}

The authors gratefully acknowledge the partial funding of Project NORTE-01-0145-FEDER-000022-SciTech-Science and
Technology for Competitive and Sustainable Industries, cofinanced by Programa Operacional Regional do Norte (NORTE2020), through Fundo Europeu de Desenvolvimento Regional (FEDER) and the FCT-Foundation for Science and Technology under the project (PEst-OE/EME/LA0022/2013).

\section{CONFLICT OF INTEREST}

There is no conflict of Interest.

\section{ORCID}

R. Vardasca (iD http://orcid.org/0000-0003-4217-2882

\section{REFERENCES}

1. Apalla Z, Nashan D, Weller RB, Castellsagué X. Skin cancer: epidemiology, disease burden, pathophysiology, diagnosis, and therapeutic approaches. Dermatol Ther (Heidelb). 2017;7:5-19. https://doi. org/10.1007/s13555-016-0165-y.

2. Brunssen A, Waldmann A, Eisemann N, Katalinic A. Impact of skin cancer screening and secondary prevention campaigns on skin cancer incidence and mortality: a systematic review. J Am Acad Dermatol. 2017;76:129-139.e10. https://doi.org/10.1016/j. jaad.2016.07.045.

3. Kolarsick PAJ, Kolarsick MA, Goodwin C. Anatomy and physiology of the skin. J Dermatol Nurses Assoc. 2011;3:203-213. https://doi. org/10.1097/JDN.0b013e3182274a98.

4. Pirtini C Cetingül M, Herman C. Quantification of the thermal signature of a melanoma lesion. Int J Therm Sci. 2011;50:421-431. https://doi.org/10.1016/j.ijthermalsci.2010.10.019.

5. Ring EFJ, Ammer K. Infrared thermal imaging in medicine. Physiol Meas. 2012;33:R33-R46. https://doi.org/10.1088/0967-3334/33/ 3/R33.

6. Wilson SB, Spence VA. A tissue heat transfer model for relating dynamic skin temperature changes to physiological parameters. Phys Med Biol Phys Med Biol. 1988;33:895-912.

7. Kaczmarek M, Nowakowski A. Active dynamic thermography in medical diagnostics. In: Ng EY, Etehadtavakol M, eds. Application of Infrared to Biomedical Sciences. Series in BioEngineering. Singapore: Springer Singapore; 2017:291-310. https://doi. org/10.1007/978-981-10-3147-2.

8. Pirtini Çetingül M, Herman C. The assessment of melanoma risk using the dynamic infrared imaging technique. J Therm Sci Eng Appl. 2011;. https://doi.org/10.1115/1.4004424.

9. Santa Cruz GA, Bertotti J, Marín J, et al. Dynamic infrared imaging of cutaneous melanoma and normal skin in patients treated with BNCT. Appl Radiat Isot. 2009;67(7-8 SUPPL.):54-58. https://doi. org/10.1016/j.apradiso.2009.03.093.

10. Herman C, Pirtini Cetingul M. Quantitative visualization and detection of skin cancer using dynamic thermal imaging. J Vis Exp. 2011;51:e2679. https://doi.org/10.3791/2679.

11. Liberati A, Altman DG, Tetzlaff J, et al. The PRISMA statement for reporting systematic reviews and meta-analyses of studies that evaluate health care interventions: explanation and elaboration. PLoS Med. 2009;6:e1000100. https://doi.org/10.1371/journal. pmed.1000100.

12. Moher D, Liberati A, Tetzlaff J, Altman D; The PRISMA Group. Preferred reporting items for systematic reviews and metaanalyses: the PRISMA statement. PLoS ONE. 2009;6:1-6. https:// doi.org/10.1371/journal.pmed.1000097. 
13. Godoy SE, Ramirez DA, Myers SA, et al. Dynamic infrared imaging for skin cancer screening. Infrared Phys Technol. 2015;70:147-152. https://doi.org/10.1016/j.infrared.2014.09.017.

14. Hashemiyan M, Valipoori Goodarzi F, Haddadnia J. Diagnosis of malignant melanoma based on tissue changes in spatial thermography images. J Dermatology Cosmet. 2016;6:221-226.

15. Stringasci MD, Moriyama LT, Salvio AG, Bagnato VS, Kurachi C. Thermographic diagnostics to discriminate skin lesions: a clinical study. In: Kurachi C, Svanberg K, Tromberg BJ, Bagnato VS, eds. Biophotonics South America Proc. of SPIE Proc. of SPIE, Vol 9531. 2015. https://doi.org/10.1117/12.2180967.

16. Di Carlo A, Elia F, Desiderio F, Catricalà C, Solivetti FM, Laino L. Can video thermography improve differential diagnosis and therapy between basal cell carcinoma and actinic keratosis? Dermatol Ther. 2014;27:290-297. https://doi.org/10.1111/ dth.12141.

17. Solivetti FM, Desiderio F, Guerrisi A, et al. HF ultrasound vs PET-CT and telethermography in the diagnosis of In-transit metastases from melanoma: a prospective study and review of the literature. J Exp Clin Cancer Res. 2014;33:96. https://doi.org/10.1186/ s13046-014-0096-3.

18. Laurino C, Palmieri B. Wide instrumental screening in monitoring early melanoma. Eur J Oncol. 2015;20:41-52.

19. Laino L, Elia F, Desiderio F, et al. The efficacy of a photolyase-based device on the cancerization field: a clinical and thermographic study. J Exp Clin Cancer Res. 2015;34:84. https://doi.org/10.1186/ s13046-015-0203-0.

20. Li J, Guo C, Wang Z, Gao K, Shi X, Liu J. Electrical stimulation towards melanoma therapy via liquid metal printed electronics on skin. Clin Transl Med. 2016;5:21. https://doi.org/10.1186/ s40169-016-0102-9.

21. Cholewka A, Stanek A, Kwiatek S, et al. Proposal of thermal imaging application in photodynamic therapy-preliminary report. Photodiagnosis Photodyn Ther. 2016;14:34-39. https://doi. org/10.1016/j.pdpdt.2015.12.003.

22. Bonmarin M, Le Gal F-A. Lock-in thermal imaging for the earlystage detection of cutaneous melanoma: a feasibility study. Comput Biol Med. 2014;47:36-43. https://doi.org/10.1016/j. compbiomed.2014.01.008.

23. Bhowmik A, Repaka R, Mishra SC. Thermographic evaluation of early melanoma within the vascularized skin using combined non-Newtonian blood flow and bioheat models. Comput Biol Med. 2014;53:206-219. https://doi.org/10.1016/j. compbiomed.2014.08.002.

24. Bhowmik A, Repaka R, Mulaveesala R, Mishra SC. Suitability of frequency modulated thermal wave imaging for skin cancer detection-a theoretical prediction. J Therm Biol. 2015;51:65-82. https:// doi.org/10.1016/j.jtherbio.2015.03.007.

25. Agyingi E, Wiandt T, Maggelakis S. A quantitative model of cutaneous melanoma diagnosis using thermography. In: Bélair J, Frigaard IA, Kunze H, Makarov R, Melnik R, Spiteri RJ, eds. Mathematical and Computational Approaches in Advancing Modern Science and Engineering. Cham: Springer International Publishing; 2016:167-175. https://doi.org/10.1007/978-3-319-30379-6_16.

26. Cheng TY, Herman C. Analysis of skin cooling for quantitative dynamic infrared imaging of near-surface lesions. Int J Therm Sci. 2014;86:175-188. https://doi.org/10.1016/j.ijthermalsci.2014. 06.033.

How to cite this article: Magalhaes C, Vardasca R, Mendes J. Recent use of medical infrared thermography in skin neoplasms. Skin Res Technol. 2018;00:1-5. https://doi.org/10.1111/srt.12469 